\section{Popovic says OSI is wrong}

SIR - In response to the articles "OSI finds Popovic guilty on Science paper"1 and "More on Gallo and Popovic", reporting that the Office of Scientific Integrity (OSI) of the National Institutes of Health (NIH) has found me guilty of scientific misconduct in connection with the 4 May 1984 Science paper of which I am first author ${ }^{3}$, I should like to say that OSI is wrong on all counts.

Six of the eight proposed 'findings' of scientific misconduct were related to individual entries of "ND" in Tables 1 and 2 , and rest on a definition in the legend to Table 1 that I do not recall writing. OSI claims that the "NDs" misrepresented our data because the legend to Table 1 defines "ND" as "not done", whereas laboratory notebooks indicate that immunofluorescence (IF) assays actually had been performed. I used "ND", however, to mean "not determinable" when unambiguous, quantitative evaluation of a slide was not possible. If I had meant to indicate that no assay was performed, I would have used the notation "NT" for "not tested", as I had done in previous publications.

Moreover, the qualitative " $+", "+/-"$ and "-" data in the notebooks, if reflected in the publication, would not have altered the conclusions. For example, in Table 2, recorded data for two HIV-1 isolates indicate that an assay using human serum (ET) was negative in one experiment but positive in another, parallel one. Moreover, contemporaneous assays using serum from patient Bru, kindly provided by Luc Montagnier, showed that 12 out of 14 IF tests exhibited clear-cut positivity, ranging from 35 to 90 per cent. (The results of assays using Bru serum were not included in the Science paper because Robert Gallo planned to publish them together with Luc Montagnier and his co-workers.)

OSI also claims that "ND" should not be used without explaining why it is impossible to provide more precise data. Scientific journals, however, often contain articles in which "ND" is used without explanation in place of quantitative information, and often no definition of "ND" is provided in the reported results. (For example, the Journal of Immunology instructs authors not to define "ND" because the journal adopts the "standard abbreviation" of "ND, not determined".)

OSI also claims that the entry " 10 per cent" positive cells for $\mathrm{H} 35$ in Table 1 was inconsistent with Elizabeth ReadConnole's laboratory notebook entries and was therefore false. But OSI misinterpreted the entries. Once ReadConnole became aware of OSI's error

1. Nature 357, 3 (1992). (through press accounts), she provided the following written statement to my attorneys and NIH officials: "My statement 'very few cells' was a comment on the number of the cells on the slide .... In my second statement, I was noting that even though there were 'very few cells', the cells that were on the slide were positive for the rabbit polyclonal antisera raised against the HIV virus ....I was never asked [by OSI] what I meant by these two statements ...."

OSI also stated that it was improper for me to supply a specific number based on my own independent reading of the IF slides. It was my practice, however, always to read the slides to verify my technician's reading.

OSI claims that the statement in the methods section that "The concentrated fluids were first shown to contain particle-associated RT" misrepresents the experiment actually performed. Robert Gallo confirms that I did not write that sentence and OSI admits it has no idea who did; it first appeared in the last draft of the manuscript and resulted from someone else's editing. OSI holds me responsible nonetheless for failing to notice the change or its implications in galley proofs.

Finally, as NIH has acknowledged, the minor discrepancies do not affect the basic findings of the paper and, despite recent press accounts to the contrary, the review of OSI's proposed report is not yet complete. As they say in America, "It's not over until it's over."

\section{Mikulas Popovic}

9917 Holmhurst Road,

Bethesda, Maryland 20817, USA

2. Nature 357, 107 (1992)

3. Science 224, 497-500 (1984).

\section{Fetal tissue banks}

SIR - Your News story "Researchers reject tissue banks" (Nature 357, 267; 1992) quotes Yale researchers who extrapolated data I supplied to Senator Orrin Hatch and that he and Senator Edward Kennedy used in a Senate debate on fetal tissues research on 31 March 1992. Unfortunately, the researchers erred in their computation.

The data Congress received come from a study of miscarriages conducted at three Manhattan hospitals between 1977 and 1981: I examined more than 3,500 normal, well-preserved specimens up to 28 weeks gestational age, and concluded that about 7 per cent of them would have been potentially suitable for transplant research.

Readers should be aware that the Yale research refers only to Parkinson's disease, in which interest focuses mainly on fetal brains of a developmental age of 7-12 weeks - a small fraction of the potentially useful fetal material. Journalists who omit this detail mislead the reader, but the researchers themselves compound the difficulty by understating by half even the amount of material available for Parkinson's-related transplant research. The Yale calculation, which you report, that "usable abortions occur, on the average, only 1.4 times a year at each hospital" is an error; in fact, about twice that number would be available for the limited purpose of Parkinson's research.

Our study remains the largest and most systematic inquiry so far into the pathology of miscarriages. The results indicate that enough tissue could be obtained to make the proposed tissue banks worthwhile (J. Byrne et al. Teratology 32, 297-315; 1985). Just how much, and under what conditions, would be determined by the new tissue bank programme.

Not everyone regards the tissue bank issue from a political perspective and, if it were taken out of politics, many researchers would support such a programme. Research using fetal tissue holds enormous promise, not only for transplantation, but also in cancer research, in developmental biology and in AIDS research.

Moreover, we are still far from understanding the causes of miscarriages, despite their common occurrence: there were an estimated 750,000 in the United States last year. Ectopic pregnancies are on the rise, yet their causes are still obscure. Much good will come from the wider availability of fetal tissue for research. The opportunity to use the tissue bank networks for new studies of fetal loss should not be lost.

\section{Jullanne Byme}

Boyne Research Foundation, Washington, DC 20010, USA

\section{Growth of science}

SIR - Edward J. Krowitz claims that the developed nations must aim not only for the absolute growth of their science, but also for relative growth with respect to developing countries (Nature 358, 186; 1992). At present, the rich countries of the West and North publish some 20 times as many papers per capita as do the poor countries of Africa and Asia, yet Krowitz wants to see this inequality widen even more.

\section{Terence Kealey}

University of Cambridge,

Department of Clinical Chemistry,

Addenbrooke's Hospital,

Hills Road, Cambridge CB2 2QR, UK 